\title{
Volume Bragg Gratings in PTR glass - New Optical Elements for Laser Design
}

\author{
Leonid Glebov \\ CREOL, The College of Optics and Photonics, University of Central Florida, P.O. Box 162700, Orlando, Florida 32816-2700 \\ Tel: (407) 823-6983 Fax: (407) 823-6880 Email: lbglebov@creol.ucf.edu
}

\begin{abstract}
This is a survey of achievements in semiconductor, solid state and fiber lasers enabled by the use of new optical elements which are volume Bragg gratings recorded in a photo-thermorefractive (PTR) glass.

(C)2008 Optical Society of America

OCIS codes: (050.7330) Volume holographic gratings; (140.3510) Lasers, fiber.
\end{abstract}

\section{Introduction}

This paper is a survey of recent achievements at the College of Optics and Photonics/CREOL at the University of Central Florida and at OptiGrate Corporation in the development of diffractive optical elements which are volume Bragg gratings recorded in a photo-thermo-refractive (PTR) glass for dramatic improvement of parameters of different types of lasers demonstrated by a number of different research groups. This new technology enabled recording of high efficiency phase volume holograms in an optical quality silicate glass. These holograms are used for creation of a number of unique diffractive optical elements that provide new opportunities for sensing, signal processing and laser beam control. The same type of glass can be used for design of conventional optical elements and windows working in high power laser beams and under high level of ionizing radiation. Moreover, this material could be used for complex elements creation such as all-solid-state lasers and combined refractive and diffractive elements.

Multiple conventional photosensitive materials for volume hologram recording have its own merits, combined with considerable drawbacks [1]. Finally, none of those materials had a combination of high phase sensitivity, low loses and high tolerance to laser radiation which are necessary for wide application in laser design. Availability of diffractive optical elements fabricated from PTR glass changed the situation.

\section{Photo-thermo-refractive glass}

The history of the study of photo-thermo-refractive process and its parameters from the point of view of hologram recording were summarized in surveys [2, 3]. Recent improvements of PTR technology [4, 5] resulted in creation of volume phase holograms (Bragg gratings) with low losses and extremely high diffraction efficiency exceeding 99\%.

PTR glass is a $\mathrm{Na}_{2} \mathrm{O}-\mathrm{ZnO}-\mathrm{Al}_{2} \mathrm{O}_{3}-\mathrm{SiO}_{2}$ glass doped with silver, cerium, and fluorine. The window of transparency ranges from 350 to $2700 \mathrm{~nm}$. Thermal variations of refractive index in PTR glass are very low $\left(\mathrm{dn} / \mathrm{dT}=5 \times 10^{-8} 1 / \mathrm{K}\right)$. The maximum value of the refractive index decrement after exposure to UV radiation and thermal development is about $10^{-3}$. The photosensitivity of PTR glass for $325 \mathrm{~nm}$ irradiation followed by 3 hours of development at $520^{\circ} \mathrm{C}$ is $1.5 \times 10^{-3} \mathrm{~cm}^{2} / \mathrm{J}$ which means that a standard dosage for high efficiency hologram recording does not exceed few hundreds of $\mathrm{mJ} / \mathrm{cm}^{2}$. That fact that refractive index modulation in PTR glass is resulted from thermal precipitation of a crystalline phase, leads to an important consequence. There is no way to destroy crystalline particles of $\mathrm{NaF}$ in glass matrix by any type of radiation. This is why PTR holograms are stable under exposure to IR, visible, UV, Xray, and gamma-ray irradiation.

\section{Volume Bragg gratings}

Bragg gratings in PTR glass were mainly recorded by an exposure to the interference pattern of radiation of He-Cd laser operating at $325 \mathrm{~nm}$ with average power of $35 \mathrm{~mW}$. Spatial frequency of gratings was varied from 50 to about $10,000 \mathrm{~mm}^{-1}$. Volume gratings in both transmitting and reflecting mode were recorded with thickness ranged from $0.5 \mathrm{~mm}$ to $25 \mathrm{~mm}$. Both transmitting and reflecting gratings could be tilted inside a glass wafer for almost arbitrary angles. Maximum aperture of gratings was up to $50 \mathrm{~mm} \times 50 \mathrm{~mm}$. Diffraction efficiency was measured at different wavelengths ranged from 355 to $2000 \mathrm{~nm}$. In all cases, maximum absolute diffraction efficiency of PTR Bragg gratings exceeded $95 \%$ while relative diffractive efficiency was demonstrated above $99.9 \%$. 


\section{MD1.pdf}

becomes narrower with increasing of spatial frequency and thickness of grating. It is important to note that angular selectivity below $1 \mathrm{mrad}$ can be achieved for Bragg gratings with thickness of several millimeters and spatial frequency exceeding several hundreds of lines per millimeter. Variations of the spatial frequency available in PTR gratings provide narrowing of the value of angular selectivity down to about $0.1 \mathrm{mrad}$ or increasing it up to several milliradians. Spectral selectivity of transmitting grating can be narrowed down to subnanometer region. For reflecting PTR gratings, deflection angles could be ranged from 120 to $180^{\circ}$, angular selectivity from 10 to $100 \mathrm{mrad}$, spectral selectivity from 0.01 to $2 \mathrm{~nm}$.

\section{Spectral selection in resonators}

The most obvious application of VBGs as dispersive elements in laser resonators for spectral narrowing and stabilization is determined by their narrow spectral selectivity combined with low losses. Such lasers which utilize VBGs were named volume Bragg lasers.

\subsection{Narrowing of emission spectra of semiconductor diodes, bars and stacks}

The use of reflecting Bragg gratings as output couplers in external resonators for semiconductor diodes, bars and stacks were described in a great number of publication, e.g. [7-10]. It was found that PTR output couplers with thickness from parts to few millimeters provide efficient spectral locking of laser diodes at the level of from 60 to $100 \mathrm{pm}$. Emission wavelength does not depend on temperature of a diode. Efficiency of a volume Bragg laser diode usually exceeds $95 \%$ of efficiency of an original diode at power level of several watts. Locking of diode bars and stacks with total power up to $0.5 \mathrm{~kW}$ were demonstrated with efficiency above $90 \%$. It was found that proper selection of grating parameters allows locking without collimation. From the opposite side, a combination of fast axis collimator and Bragg output coupler in the same piece of glass was successfully demonstrated [11-12].

\subsection{Extra narrow high power semiconductor lasers}

A new challenge for spectral narrowing was generated by resent development of diode pumped alkali lasers [13]. To satisfy requirements for efficient absorption of diode radiation by low pressure Rb-cells a volume Bragg diode laser was developed on the basis of VBG with thickness exceeding $10 \mathrm{~mm}$ which demonstrated up to $2 \mathrm{~W}$ CW power within $20 \mathrm{pm}$ at $780 \mathrm{~nm}$ [14]. Recent extension of this approach to a diode bar has shown spectral locking of $30 \mathrm{~W}$ within $30 \mathrm{pm}$ with total efficiency more than $90 \%$.

\subsection{Narrowing of solid state lasers}

The use of PTR Bragg mirrors as output couplers in solid state lasers was described in publications [15, 16]. It was demonstrated that Bragg mirrors with thickness of several millimeters enable spectral narrowing down to couple picometers with approximately the same power as for a laser with a conventional output coupler. A combination of a thick Bragg mirror and a Fabri-Perot etalon enabled selection of single frequency in different rare earth ions doped lasers $[17,18]$.

\subsection{Spectral selection in regenerative amplifiers}

Dramatic impact was produced by PTR Bragg mirrors incorporated in Nd:YLF regenerative amplifier [19, 20]. Spectral filtering resulted in suppression of background and increasing of contrast of amplified pulse for about three orders of magnitude.

\section{Angular selection in resonators}

It is important to note that volume Bragg grating works in angular space. It means that it is not necessary to produce Fourier transform for processing with angular distribution of radiation. One of the consequences is that it is possible to produce selection of transverse modes in resonators without collimation or re-focusing (conventional spatial filtering) radiation. Experiments with wide stripe semiconductor lasers [9, 10, 21] have shown that the use of VBGs with angular selectivity comparable with diffraction limited divergence of emitter provides amplification for a single mode only. It is important that a selected mode can be a mode with arbitrary number. Close to diffraction limited divergence was obtained from laser diodes with stripe width up to $250 \mu \mathrm{m}$ at power level of several watts. This approach enables overcoming the basic restriction in laser design which is a requirement of a single Fresnel zone at an output coupler.

\section{Phase locking in multi-channel resonators}

Coherent combining or phase locking of multichannel laser devices is usually considered as a promising way for high power laser sources. A new approach to this problem was demonstrated in publications [22, 23] where extremely narrow-band PTR VBGs were used for radiation exchange between two laser diodes. It was demonstrated that coherent radiation from two laser diodes placed at separated stages could be observed for a year at driving current exceeding 4 thresholds. Recently, phase locking of three lasers was demonstrated (to be published). 


\title{
MD1.pdf
}

\section{Stretching and compression of laser pulses}

It is possible to fabricate a reflecting volume grating with a gradually varied period in the direction of beam propagation (longitudinally chirped grating). Such chirped Bragg mirror would reflect different wavelengths from different parts of such a thick mirror. This means that a femtosecond laser pulse with wide spectrum after reflection would be stretched in space. This stretched pulse being directed to the same chirped Bragg mirror from the opposite side, would be compressed back to femtoseconds. Such gratins were fabricated and experiment have shown [24, 25] that stretching and compression could be performed with efficiency of about $95 \%$. it is beneficial that laser damage threshold of a volume grating is higher than that for surface gratings and this element cannot me misaligned.

\section{Beam deflection}

The fact that dependence of diffraction efficiency of VBG on incident angle has the main maximum and a series of zeros enables the use of such gratings as angular magnifiers. Deflection of an incident beam for a small angle (it can be in milliradian range) leads to deflection of a diffracted beam for a predetermined arbitrary large angle. This phenomenon is the basis for high speed non-mechanical beam steering in high power laser systems [26] and in portable opto-electronic devices [27].

\section{Spectral beam combining}

The similar fact that dependence of diffractive efficiency of VBG on wavelength has the main maximum and a series of zeros enables the use of such gratings as beam combiners. If two laser beams with wavelengths corresponding to maximum and zero diffraction efficiency are launched to a Bragg grating at complementary Bragg angles, the first beam would be totally diffracted while the second one would be totally transmitted. This means that both these beams would propagate in the same space and in the same direction. Such approach was used for combining of beams of Yb-doped fiber lasers [28-30]. Efficiency of five-channel combining of 93\% with divergence close to diffraction limit and total power exceeding $700 \mathrm{~W}$ were demonstrated. The same approach was successfully applied for semiconductor lasers [31].

\section{Conclusion}

Development of high-efficiency, low-losses, robust diffractive elements which are volume Bragg gratings in photothermo-refractive (PTR) glass enabled remarkable progress in laser development and beam control.

\author{
References: \\ 1. P. Hariharan. Optical Holography. Principles, techniques, and applications. Cambridge University Press, 1996. \\ 2. L.B. Glebov. Photosensitive glass for phase hologram recording. Glass Science and Technology, 71C (1998) 85-90. \\ 3. L.B. Glebov. Volume hologram recording in inorganic glasses. Glass Science and Technology 75 C1 (2002) 73-90. \\ 4. O.M. Efimov, L.B. Glebov, L.N. Glebova, V.I. Smirnov. United States Patent 6,586,141 (2003). \\ 5. O.M. Efimov, L.B. Glebov, V.I. Smirnov. United States Patent 6,673,497 (2004). \\ 6. I.V. Ciapurin, L.B. Glebov, V.I. Smirnov, Optical Engineering 45 (2006) 015802, 1-9. \\ 7. B.L.Volodin, S.V.Dolgy, E.D.Melnik, E.Downs, J.Shaw, and V.S.Ban, Opt. Lett, 29, 1891 (2004). \\ 8. G. Venus, V. Smirnov, L. Glebov, and M. Kanskar. Proceedings of Solid State and Diode Lasers Technical Review. (2004) P-14. \\ 9. G.B. Venus, A. Sevian, V.I. Smirnov, L.B. Glebov. Proc. of SPIE 5711 (2005) 166-176. \\ 10. L.B. Glebov. Proc. of SPIE 6216 (2006) 621601. \\ 11. C. Schnitzler, et al. Proceedings SPIE 6456 (2006) 645612. \\ 12. Lutz Aschke, et al. Proceedings of Solid State and Diode Lasers Technical Review (2007), Diode1-1. \\ 13. W.F.Krupke, R.J.Beach, V.K.Kanz, S.A.Payne, Opt. Lett. 28, 2336 (2003). \\ 14. A. Gourevitch, G. Venus, V. Smirnov, and L. Glebov. Opt. Lett. 32 (2007) 2611-2613. \\ 15. T. Chung, A. Rapaport, V. Smirnov, L. Glebov, M. Richardson, and M. Bass. Opt. Lett. 31 (2006) 229-231. \\ 16. T. Chung, et al. Proc. of SPIE 6216 (2006) 621603. \\ 17. B. Jacobsson, V. Pasiskevicius, and F. Laurell. Opt. Lett., 31, 1663-1665 (2006). \\ 18. Bjorn Jacobsson, Valdas Pasiskevicius and Fredrik Laurell. Optics Express 14 (2006) 92849292. \\ 19. A. V. Okishev, C. Dorrer, V. I. Smirnov, L. B. Glebov, and J. D. Zuegel. Opt. Express 15 (2007) 8197-8202. \\ 20. C. Dorrer, A. V. Okishev, I. A. Begishev, J. D. Zuegel, V. I. Smirnov, and L. B. Glebov. Opt. Lett. 32 (2007) 2378-2380. \\ 21. G. Venus, L. Glebov, V. Rotar, V. Smirnov, P. Crump, and J. Farmer. Proc. of SPIE 6216 (2006) 621602. \\ 22. G. Venus, A. Sevian, V. Smirnov, L. Glebov. Opt. Lett. 31 (2006) 1453-1455. \\ 23. S. Riyopoulos, G. Venus and L. Glebov. Proc. of SPIE 6456 (2007), 645613, 1-7. \\ 24. V. Smirnov, E. Flecher, L. Glebov, K. Liao, and A. Galvanauskas. Proceedings of SSDLTR. 2005, SS2-1. \\ 25. K. Liao, M. Cheng, E. Flecher, V. Smirnov, L. Glebov, and A. Galvanauskas. Opt. Express 15 (2007) 4876-4882. \\ 26. D.P. Resler, D.S. Hobbs, R.C. Sharp, L.J. Friedman, T.A. Dorschner. Opt. Let., 21 (1996) 689-672. \\ 27. A. Glebov, A. Sugama, V. Smirnov, S. Aoki, V. Rotar, M. Lee, and L. Glebov. IEEE Photonics Technology Lett. 19 (2007) $701-703$. \\ 28. I, Ciapurin, L, Glebov, V, Smirnov. Proceedings of SPIE 5335 (2004) 116-124. \\ 29. O. Andrusyak, I. Ciapurin, V. Smirnov, G. Venus, and L. Glebov. Proc. of SPIE 6453 (2007) 64531 L1-L7. \\ 30. O. Andrusyak, V. Rotar, A. Sevian, V. Smirnov, G. Venus, and L. Glebov. Proceedings of SSDLTR (2007), BC2-2. \\ 31. Y. Kaneda, et al. IEEE Phot. Tech. Lett. 18 (2006) 1795-1797.
}

